Supporting Information for

\title{
Molecular mechanisms of diverse activation stimulated by different biased agonists for $\beta 2$ - Adrenergic Receptor
}

\author{
Jianfang Chen ${ }^{a}$, Jiangting Liu ${ }^{a}$, Yuan Yuan ${ }^{b}$, Xin Chen ${ }^{a}$, Fuhui Zhang ${ }^{a}$,
}

\section{Xuemei $P u^{a}$ *}

${ }^{a}$ College of Chemistry, Sichuan University, Chengdu 610064, China

${ }^{\mathrm{b}}$ College of Management, Southwest University for Nationalities, Chengdu 610041,

China 


\section{Simulation2}

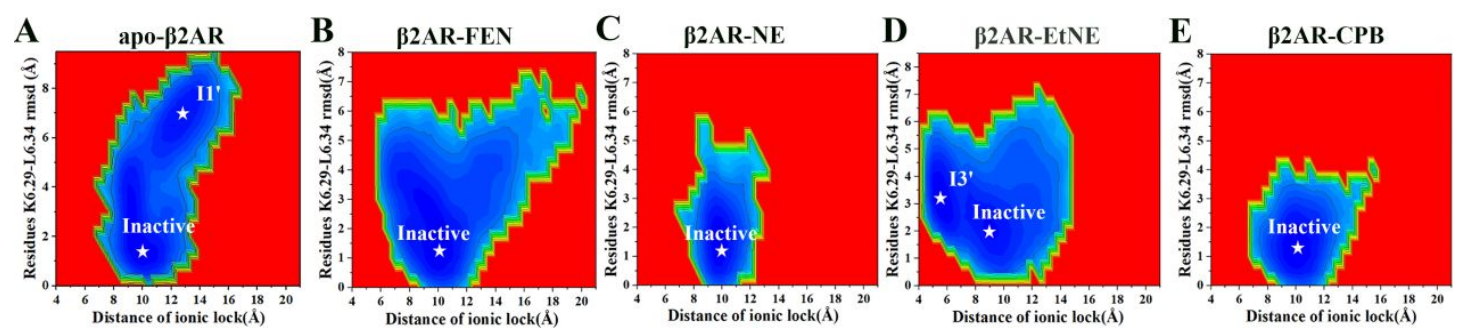

Figure S1. Two-dimensional PMF profiles of the distance of ionic lock (R131 ${ }^{3.50} \mathrm{Ca}-$ E268 ${ }^{6.30} \mathrm{C} \alpha$ ) and RMSD of backbone atoms of residues at the TM6 cytoplasmic end (K267 $7^{6.29}-\mathrm{L} 272^{6.34}$ ) with respect to the inactive crystal structure for the second parallel simulation trajectories of the apo- $\beta 2 \mathrm{AR}(\mathrm{A})$ and the $\beta 2 \mathrm{AR}$ bound by the G-proteinbiased agonist FEN (B), the balanced agonist NE (C), and $\beta$-arrestin-biased agonists EtNE(D) and CPB(E). Distinct conformational states of TM6 identified in the lowenergy wells are highlighted by Pentagrams. PMF profiles in the second parallel simulation trajectories are similar to the first one shown in Figure 2. 


\section{Simulation2}

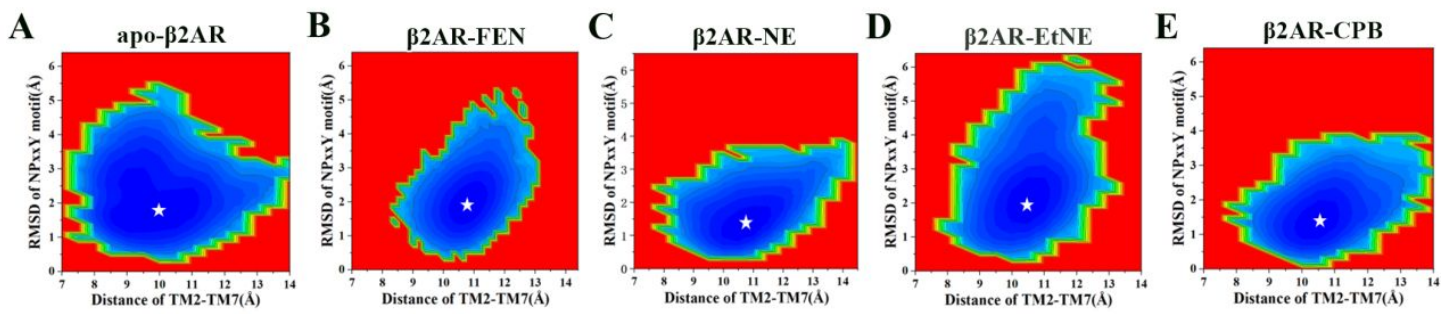

Figure S2. Two-dimensional PMF profiles of the distance between $\mathrm{L} 75^{2.46} \mathrm{Ca}-\mathrm{P} 323^{7.50}$ Ca and the RMSD of NPxxY motif (RMSD of backbone atoms of residues N3227.49 Y $326^{7.53}$ ) for the second parallel simulation trajectories of the apo- $\beta 2 A R(A)$ and the B2AR bound to the G-protein-biased agonist FEN (B) and balanced agonist NE (C) and $\beta$-arrestin-biased agonists EtNE (D) and CPB (E). The conformational states of TM7 identified in the low-energy wells are highlighted by Pentagrams. It can be seen that the results are similar between the two parallel trajectories. 


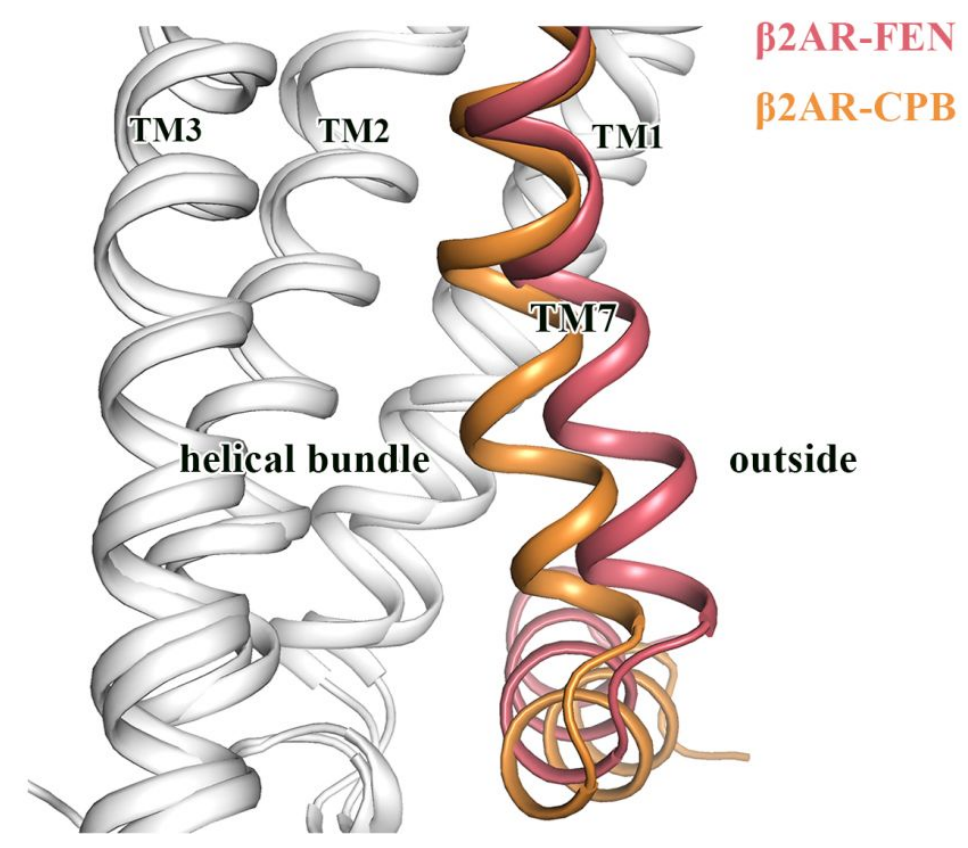

Figure S3. Superimposed structures of FEN-bound $\beta 2 A R$ (Red) and CPB-bound $\beta 2 A R$ (Orange). 
A rhodopsin-arrestin

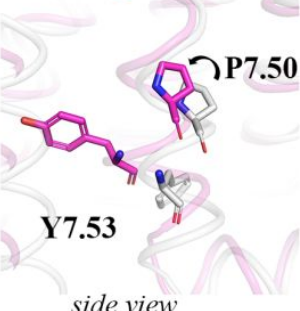

side view

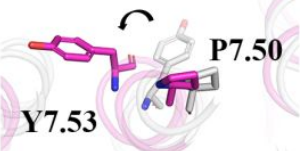

top-down view

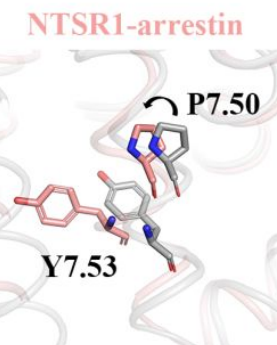

side view

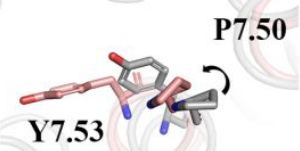

top-down view

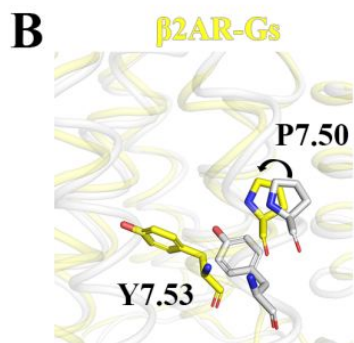

side view

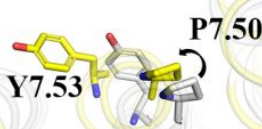

top-down view
NTSR1-Gi

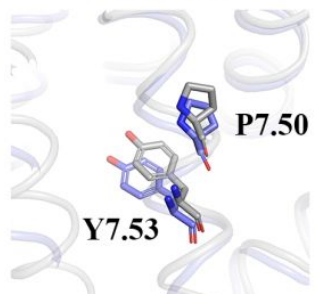

side view
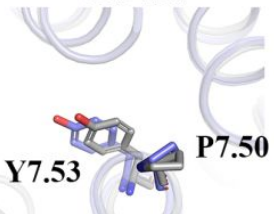

top-down view

Figure S4. Comparisons of TM7 conformations between the active crystal structures and the inactive ones for three class A GPCRs. (A) Two active crystal structures bound by arrestin. TM7 in of rhodopsin-arrestin (PDB ID: 5DGY, Magenta) and NTSR1arrestin (PDB ID: 6UP7, Pink) exhibits a counterclockwise twist (top-down view) relative to their inactive crystal structures. (B) Two active crystal structures bound by G-protein. TM7 shows two conformations: the counterclockwise twist (top-down view) for 32 AR-Gs (PDB ID: 3SN6, Yellow) and slightly counterclockwise for NTSR1-Gi (PDB ID: 6OSA, Purple) relative to their inactive crystal structures. All the inactive crystal structures are colored with white for rhodopsin (PDB ID: 1U19), NTSR1 (PDB ID: 4BV0) and $\beta 2 \mathrm{AR}$ (PDB ID: 2RH1). 


\section{Simulation2}

A

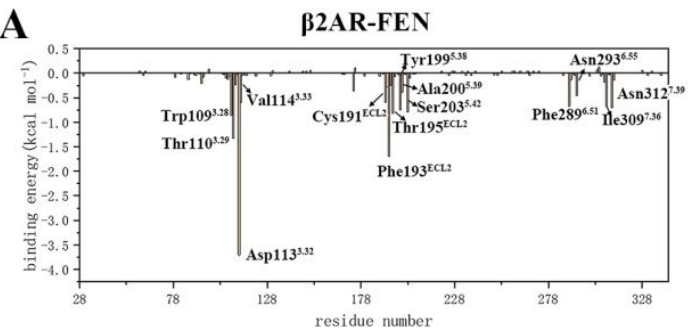

C

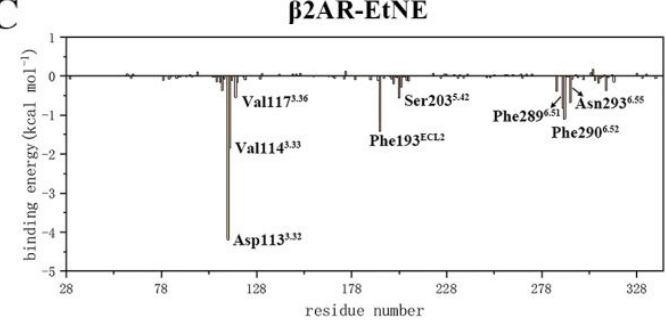

B

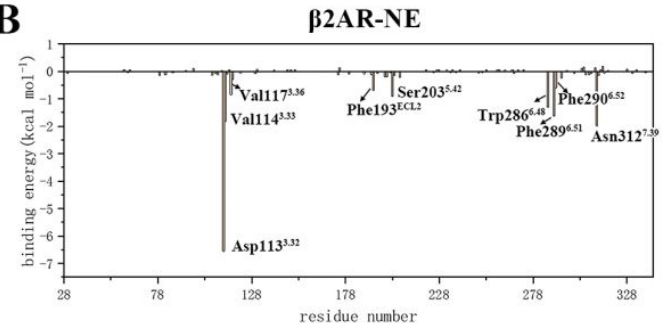

D

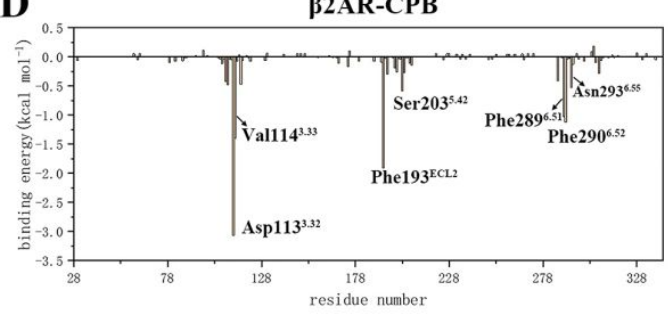

Figure S5. Residues contributing to the binding of $\beta 2 A R$ and G-protein-biased agonist

FEN (A) and balanced agonist NE (B) and $\beta$-arrestin-biased agonists $\operatorname{EtNE}(\mathrm{C})$ and $\mathrm{CPB}(\mathrm{D})$ for the second parallel trajectories. The $3 \mu$ s trajectory is taken every ten frames (the total 30,000 frames for each trajectory) to calculate the binding free energies. Residues with absolute value of binding energies greater than $0.5 \mathrm{kcal} / \mathrm{mol}$ are marked. Similar to the result from the first parallel trajectory shown in Figure 7, FEN forms interactions with more upper pocket residues in TM5 and only with one hydrophobic pocket residue Phe2896.51 on TM6. The other three agonists interact with fewer TM5 residues, but with more hydrophobic pocket residues on TM6 (Trp286 6.48 and Phe2906.52). 
A

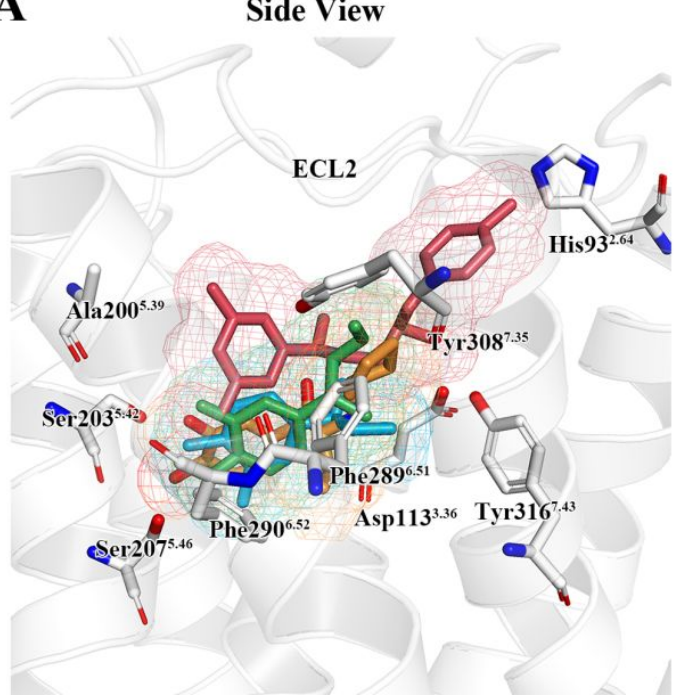

B

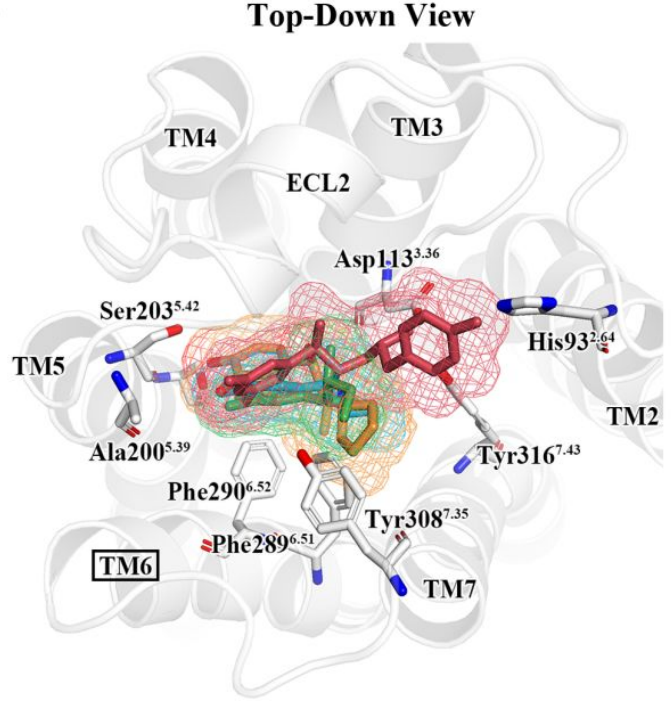

Figure S6. The superposition of the four agonists in the pocket from side view and the top-down view. Red for G-protein-biased agonist FEN, Blue for balanced agonist NE, Green and Orange for $\beta$-arrestin-biased agonists EtNE and CPB, respectively. White for the receptor. 


\section{Simulation2}

A

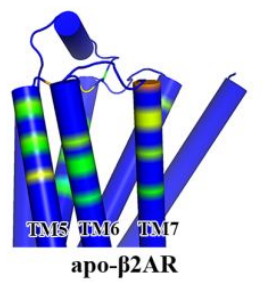

B

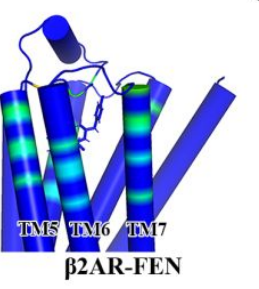

C

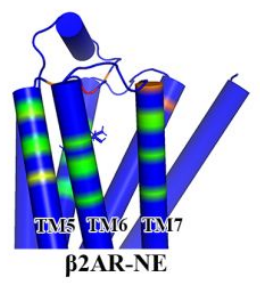

D

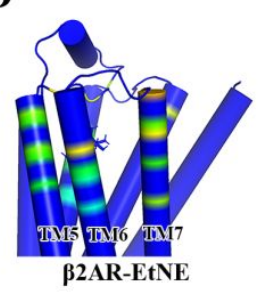

E

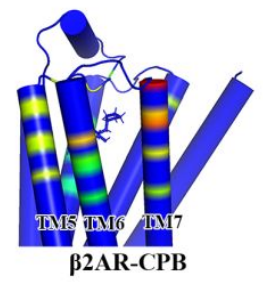

Figure S7. Schematic representation of the receptor structures colored by the RMSF of residues in the ligand binding pocket for the second parallel simulations, and the color scale is from $0 \AA$ (blue) to $2.2 \AA$ (red). Similar to the first parallel simulation shown in Figure 9, the residues of TM5 are more rigid upon binding of FEN than the other three agonists. 


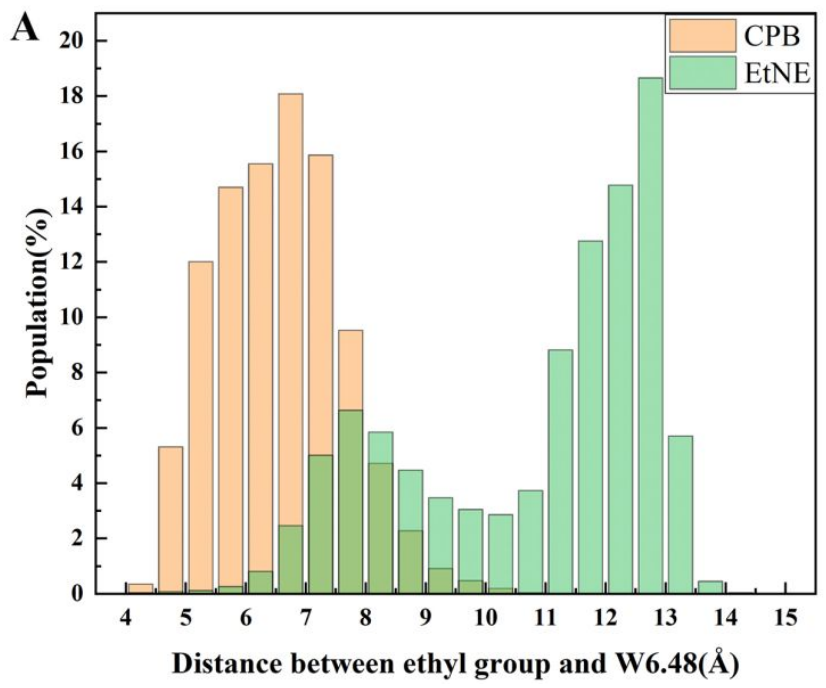

B

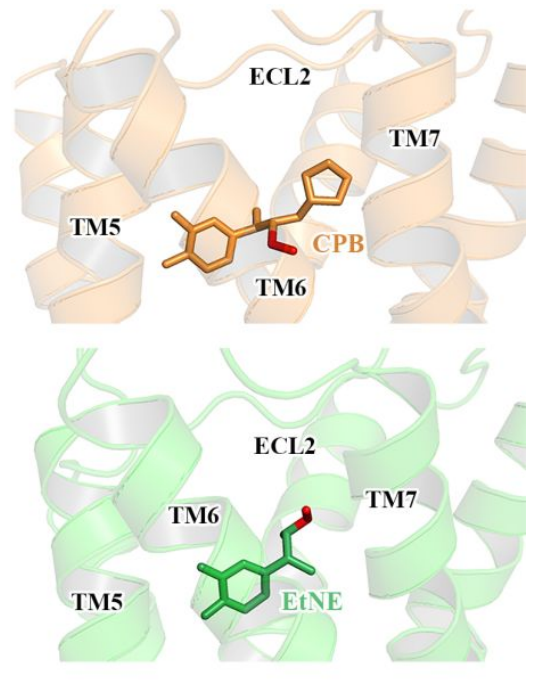

Figure S8. The distance between TM6 and the ethyl group of $\beta$-arrestin-biased agonists.

(A) The distance distributions between the ethyl group of $\beta$-arrestin-biased agonists and W6.48. (B) The representative binding poses of the $\beta$-arrestin-biased agonists EtNE and CPB. The ethyl group (red) of CPB is closer to TM6 than the ethyl group (red) of EtNE. 